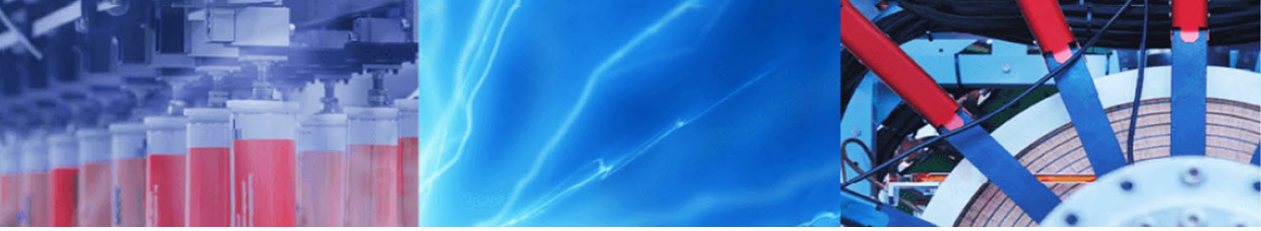

Research Article

\title{
Experimental validation of numerical predictions for "Deviant" density enhancement of protein emulsions in oil (Oleo-Nanofluids)
}

\author{
Aditya Kuchibhotla $^{1}$ (1) $\cdot$ Sanjukta Chakraborty ${ }^{2} \cdot$ Debjyoti Banerjee $^{3}$
}

Received: 9 March 2020 / Accepted: 24 August 2020 / Published online: 1 September 2020

(c) Springer Nature Switzerland AG 2020

\begin{abstract}
In this study, an analytical model for estimating the total effective density of nanofluids is developed and experimentally validated. The numerical model is based on weighted average, in proportion, to the indvidual mass fractions of solvent, nanoparticle, and compressed phase of solvent around nanoparticle. Experimental validation of the numerical predictions for deviations in density values, over that of the simple mixture rule, were performed. The neat solvent used in the experiments was a paraffin oil-based phase change material. The experimental results show that the overall enhancement in the density of the nanofluid was $10.9 \%$ of which the formation of the compressed phase can be attributed to cause the nanofluid density to deviate by $3 \%$. The "deviant" component of the enhancement for the measured values of density of the nanofluid samples are calculated by taking the difference between the measured values of density and the values predicted by the conventional mixture rule. The experimental results from this study conclusively demonstrate that the formation of the compressed phase can cause deviant behavior by anomalous enhancement of the density ("surplus density") of the samples of oleo-nanofluids synthesized in this study.
\end{abstract}

Keywords Archimedes principle - Buoyancy - Compressed phase - Surface adsorption - Phase change materials · PCM . Oleo-nanofluids · Aqueous nanofluids · Nanofin effect $\cdot \mathrm{nFE}$

\section{Introduction}

Microscopic particles with at least one dimension being less than $100 \mathrm{~nm}$ are termed as "nanoparticles". Stable colloidal suspensions of nanoparticles in a chosen solvent are often termed as "nanofluids". This topic has gained popularity in the contemporary literature due to the anomalous enhancement of the material properties of nanofluids (compared to that of the neat solvent), such as thermal conductivity and specific heat capacity. Enhancement of the material properties of nanofluids, compared to that of the neat solvents (e.g., water, glycols, oil, molten salts, etc.), have been reported extensively in the literature [1-3].
Nanofluid literature is replete with conflicting reports and controversies, based on the levels of enhancements (or degradation) in the material properties of the nanofluids that were reported by different research groups. The origin of these controversies can be traced back to the lack of due-diligence or attention to details, such as the inadequate design-of-experiments [4]. In addition, the material properties of nanofluids are often highly sensitive to minor variations in the synthesis protocols, since the stability of the nanofluids is highly sensitive to narrow range of parameters in the synthesis protocol (e.g., during batch fabrication, the synthesis protocols of nanofluids need to be developed with ardent rigor and should be adhered to diligently with adequate precision and careful monitoring

Aditya Kuchibhotla, adityak@tamu.edu; Sanjukta Chakraborty, schakraborty@tamu.edu; Debjyoti Banerjee, banerjee@tamu.edu | 'Department of Mechanical Engineering (MEEN), Texas A\&M University, College Station, TX 77843-3123, USA. ${ }^{2}$ Medical Physiology Department, College of Medicine, Texas A\&M University, Bryan, TX 77807, USA. ${ }^{3}$ Mary Kay O'Connor Process Safety Center (MKOPSC), Texas A\&M University, College Station, TX 77843-3123, USA. 
of the process variables). Hence, the early reports in the literature often neglected to rigorously control the synthesis protocol such as verifying the size and shape of nanoparticles, both before and after each experiment [4].

These anamolous deviations in material properties can arise from the spontaneous formation of a "third phase" within the bulk solvent phase that envelopes individual nanoparticles. Surface adsorption of the solvent molecules on the nanoparticle surface cause re-ordering of the molecules of the solvent phase on the surface of the nanoparticle, that can also mimic the crystal structure of the underlying solid surface [5]. Several researchers validated the presence of the third phase (also termed as the "compressed phase") by using molecular dynamics (MD) simulations and experimental investigations such as electron microscopy and X-ray diffraction measurements $[2,6]$. The compressed phase has a higher density with thickness typically ranging from 1 to $2 \mathrm{~nm}$. These literature reports also demonstrated the magnitude of the density oscillations in the compressed phase, often, with the exponentially decaying values of the peak densities in the density profile appearing to be propagating away from the surface of the nanoparticles [7].

The coupled non-linear transport mechanisms responsible for these anomalous deviations in material properties also need further exploration since the models developed in the literature are often inadequate for capturing the full spectrum of such "deviant" behavior demonstrated by all classes of nanofluids. For example, aqueous nanofluids have lower specific heat capacity values, while in contrast; non-aqueous nanofluids and oleo-nanofluids have enhanced specific heat capacity values compared to that of the neat solvent. Such anomalies can be explained to be caused by the spontaneous formation of "compressed phase" that envelopes the nanoparticles [8]. More sophisticated studies have demonstrated the formation of secondary nanostructures that emanate from the compressed phase-resulting in the formation of a percolation network of semi-crystalline phase that permeates the bulk (amorphous) solvent phase $[9,10]$. Enhancement of the specific heat capacity values was not detected in nanofluid samples that lacked the secondary nanostructure. More sophisticated numerical models are under development that can adequately predict the specific heat capacity enhancement in these nanofluids samples while accounting for the percolation networks (secondary nanostructures) that are induced in the bulk amorphous phase of the solvent [11].

\subsection{NanoFin Effect ("nFE")}

The compressed phase has also been attributed to cause peculiar phenomena-which are modeled by the "nanoFin
Effect" (" $n F E$ ") [12]. One manifestation of nFE occurs when nanoparticle coatings formed by nanoparticles cause "anomalous" thermal performance. For example, nanofins formed using nanoparticles with lower thermal conductivity values can cause higher levels of enhancement in heat flux values (compared to that of nanoparticles with higher values of thermal conductivity), especially during phasechange such as boiling on nanostructured heaters [13]. A transport model that resolves the conundrum associated with these anomalous heat transfer phenomena i.e., due to the formation of the compressed phase that envelopes these nanoparticles was therefore termed as the "nanoFin Effect" ("nFE") [12].

Prior experimental reports involving pool boiling on nanostructured heater surfaces indicated greater degree of critical heat flux (CHF) enhancement for heater surfaces coated with lower thermal conductivity nanofins compared to that of surfaces coated with higher thermal conductivity nanofins. For instance, silicon heater surfaces with etched silicon nanofins exhibited a CHF enhancement of $120 \%$ (compared to $60 \%$ enhancement in CHF observed in silicon substrates coated with carbon nanotubes/"CNT") [14]. The reason for these peculiar experimental observations is attributed to the interfacial thermal resistance (also called "Kapitza resistance" [15-17] between nanofin surface and surrounding solvent molecules resulting from impedance mismatch of the thermo-mechanical vibrations between nanofin surface and solvent molecules [18]. Hence, the higher levels of enhancement for heat transfer involving silicon nanofins is a result of their lower interfacial resistance compared to that of the CNT coated heaters by three orders of magnitude [19-21]. Therefore, the lower values of the total thermal resistance of the silicon nanofins compared to that of the CNT, including the Kapitza resistance, leads to the higher levels of enhancement in heat flux values for silicon nanofins. Consequently, the Kapitza resistance is the dominant component of the total thermal resistance in the thermal impedance network of these nanofins $[7,22,23]$.

Furthermore, by employing molecular dynamics (MD) simulations, Jo [7] investigated the effects accruing from the formation of the "compressed phase" of solvent molecules around various nanoparticles (e.g., SWNT, graphite sheets, and $C_{60}$ Fullerene) in a high temperature salt eutectic. Simulation results indicated that the thickness of the compressed layer around the nanoparticle was weakly sensitive (or almost insensitive) to the size or shape of the nanoparticle, for a given combination of solid and fluid (solvent) materials. In addition, the spatial variation of concentration (e.g., due to Van Der Waals forces and ionic force interactions within the solvent and in the vicinity of nanofins) was shown to affect the thermo-physical properties of the nanoparticle mixture. The results indicated the 
existence of oscillations in the profiles for density and ionic concentrations in the solvent phase due to the presence of a graphite nanoparticle. These oscillations would cause severe variations in species concentration gradients at different distances away from the surface of a nanoparticle. These estimates were obtained from MD simulations using classical force field models [7]. The plots-for the spatial distribution (in the radial direction) of the density and species concentration profiles-demonstrated the existence of the compressed phase where a region in the solvent phase has higher magnitudes of density in the immediate vicinity of the nanoparticle surface. The MD simulations also showed that the thickness of this compressed phase is in the range of 1-2 $\mathrm{nm}$ [7] and a "void space" between the solvent molecules and the nanoparticle surface. The thickness of the void space on the nanoparticle surface (where no solvent molecules exist) is approximately $\sim 5 \AA$ $(\sim 0.5 \mathrm{~nm})$. This void space corresponds to the equilibrium spacing (average intermolecular spacing) between the solvent molecules and the atoms on the surface of the nanoparticle.

Yang [24] investigated the implications of the compressed layer (i.e., density and concentration gradients) on the transient heat transfer in nanoparticle suspensions as well as for heaters with nanostructured surfaces. The local variations in the thermal characteristics resulting from the different values of the thermal properties of the bulk phase and the compressed phase can be attributed to the surface adsorption of the fluid molecules on the solid surfaces. The density profile was observed near a $\mathrm{SiO}_{2}$ nanoparticle immersed in a refrigerant (PF5060, which was the working fluid for the pool boiling experiments in this study [24]). Consistent with prior literature reports [25-27], Yang [24] observed that the compressed layer thickness was $\sim 1 \mathrm{~nm}$ and the peak density of the compressed phase was predicted to be that of solid phase density of solvent (i.e., approximately twice as much as the bulk phase values). Furthermore, the formation of a compressed phase of the solvent molecules around the nanoparticles or nanofins was found to be the dominant mechanism responsible for enhancing the effective specific heat capacity of the nanocomposites (e.g., nanoparticles suspended in a solvent) [28]. In other words, the formation of the compressed phase, due to the surface adsorption and the resulting reordering of the solvent molecules on the nanoparticle (or nanofin)-can provide a supplementary receptacle for additional thermal energy storage.

While the density fluctuations near the solid-liquid interface of a nanofin or a nanoparticle, can have direct implications on the thermo-physical properties (e.g., thermal conductivity and specific heat capacity), nFE implies that severe concentration gradient values caused by the concomitant oscillations in species concentration also has a significant impact on the transport mechanisms. The formation of a concentration gradient of the solvent molecules on the nanoparticle (or nanofin) surface results in a bias that modulates the heat flux depending on the directionality of the temperature difference (or "diode" like behavior for material and thermal transport), i.e., according to the predictions afforded by the $n F E$ model, it causes the formation of a thermal diode [24]. The mass transfer (or diffusion) resulting from the chemical concentration gradient drives this thermal diode like behavior where higher heat flux is achieved for a hot nanoparticle to colder solvent phase (where the thermal gradient and species concentration gradients are covariant, i.e., they decay in the same direction), than that of the configuration involving a cold nanoparticle in a hot solvent phase (where the thermal gradient and species concentration gradients are contravariant, as they decay in the opposite directions). Hence, for the same temperature difference between two nanoparticles and the liquid phase, a hot nanoparticle (or nanofin) is expected to yield $2-10 \%$ higher heat flux than that of a cold nanoparticle (depending on the material combination chosen for these experiments).

To summarize, the addition of nanoparticles into a neat solvent (or surface modification through formation of nanostructures or nanofins on heater surfaces) often result in anomalous enhancement in specific heat capacity as well as thermal conductivity while also causing deviant enhancement of heat flux values; as observed from the varying levels of enhancement of phase-change heat transfer (such as boiling) and is termed as the "nano-Fin Effect" ("FE)" [12]. This phenomenon ( $n F E$ ) arises due to non-linear coupling of the transport mechanisms involving temperature gradients and mass concentration gradients (as well as species concentration gradients). The authors [29] proposed a combination of transport mechanisms, which are primarily dominated by three distinct transport mechanisms, itemized as follows:

1. Surface adsorption of solvent molecules around the nanoparticle resulting in the formation of a compressed phase ("nanolayer" with a typical thickness of 1-2 $\mathrm{nm}$ on the solid surface) with different effective mass density and specific heat capacity than that of the bulk solvent phase (thus contributing to the thermal resistance and thermal capacitance of the mixture);

2. Interfacial thermal resistance between the nanoparticle and solvent molecule (termed as "Kapitza resistance") resulting from the vibration impedance mismatch (for the molecular and atomic scale vibrations arising from the thermal transport) between the solvent molecules and atoms on the surface of the nanoparticles; 
3. Mass diffusion driven by the concentration gradients resulting from the preferential adsorption of different species of the solvent on the surface of the nanoparticles, i.e., adhesion of the solvent species on the surface of the nanoparticle (this in turn causes gradients in the mass concentration and gradients in the ionic concentration of the different species in the solvent phase, this contributing to the formation of a "thermal diode").

It is interesting to note that $n F E$ is most pronounced for the following situations or combination of situations [12]: (i) for nanoparticle diameters less than $10 \mathrm{~nm}$ (and particularly acute for nanoparticle diameters less than $6 \mathrm{~nm}$ ), (ii) for nanoparticle density values less than $1 \mathrm{~g} /$ cc; (iii) for solvents with solid phase density higher than that of the liquid phase; (iv) for solvents with liquid phase density less than $1 \mathrm{~g} / \mathrm{cc}$; $(\mathrm{v})$ for solvents with solid phase density greater than $1 \mathrm{~g} / \mathrm{cc}$; (vi) for solvents where the ratio of liquid phase density to that of the solid phase density is less than 1; and (vii) for the material combinations (i.e., the combination of nanoparticle and solvent) where the thickness of the compressed phase is maximized, such as through control of $\mathrm{pH}$ or Zeta potential (for ionic fluids) or surfactant concentration(for polar fluids). This shows that the consequences of $\mathrm{nFE}$ are very hard to detect (to almost non-existent) for aqueous nanofluids and more pronounced for non-aqueous solvents (e.g., oleo-nanofluids, molten salt nanofluids, etc.).

Compared to the numerous literature reports on thermal conductivity of nanofluids (and to a lesser extent, the literature reports on the specific heat capacity of nanofluids), surprisingly, only a few reports [30-32] were detected, from our literature review, that have consciously attempted to perform experimental measurements for the values of the density of nanofluids, albeit erroneously. Of these experimental measurements reported in the literature, only six [30-35] have pursued consciously (or hypothesized that) the underlying causes for deviations in density enhancement were a result of the formation of compressed layer (sometimes referred to in these literature reports as the "nanolayer"). Interestingly, there was one study [30] that assumed that the simple mixing rule was valid and proceeded to suggest a correction factor, even though the surplus density exceeded $8 \%$ in some of the nanofluids samples used in their experimental measurements.

In majority of the studies reported in the literature, classical or simple mixing model for density (i.e., linear approach for estimating density values and volume fractions, based on thermodynamic equilibrium) was used for the nanofluids. As mentioned before, only a few of these studies consciously accounted for the contribution of the enhanced density of the compressed phase in the nanofluid in modulating the net density of the mixture [34, 35], but suffered from severe deficiencies, itemized below:

(a) Measurement uncertainty was not reported or measurement uncertainty analyses were faulty and completely erroneous. For example, one study erroneously claimed that measurement uncertainty for density measurement was $0.012-0.016 \%$ [35], which clearly shows that the authors misunderstood the procedure for estimating measurement uncertainty (as repeatability of the experiments was not demonstrated and the statistical uncertainty values for the measurements were not obtained).

(b) Many studies reported increase in deviant density component with increasing mass fraction of the nanoparticles. However, the theoretical underpinning was not explored or validated [30].

(c) In all studies, except one [32], the diameter of the nanoparticles was more than $10 \mathrm{~nm}$, often in the range of $40-60 \mathrm{~nm}$. As will be shown later in this study, that the deviant density component is expected to be below the measurement uncertainty values for nanoparticle diameter less than $10 \mathrm{~nm}$. Only one study [32] reported density measurement in aqueous nanofluid, with alumina nanoparticle diameter less than $10 \mathrm{~nm}$ and deviant density of $6 \mathrm{mg} / \mathrm{cc}$ or $0.6 \%$ where the precision in the measurement apparatus was reported to be $0.1 \mathrm{mg} / \mathrm{cc}$.

(d) In all studies, the density of the nanoparticles was more than $1 \mathrm{~g} / \mathrm{cc}$, and often the density of the nanoparticles was in the range of 2-6 g/cc [30,33]. As will be shown later in this study, that the deviant density component is expected to be conclusively above the measurement uncertainty values (with statistical significance greater than 68\%) for nanoparticle density less than $1 \mathrm{~g} / \mathrm{cc}$.

(e) Some of the studies involved nanoparticle mass fractions greater than $5 \%$. These results are circumspect as agglomeration kinetics dominate and compromise the stability of the nanoparticles for higher mass fractions and often it is desirable to perform the experiments for nanoparticle mass fractions to be less than $2 \%$ (and ideally $1 \%$ or less).[30, 34]

(f) Two sets of measurement instruments were used for the same measurement but only one set of measurements for density were reported [33]. Therefore, it is not clear if there were discrepancies between the two sets of measurements.

(g) One study showed deviant density component caused the specific heat capacity of the aqueous nanofluid to be conspicuously lower than that of the solvent [33], and violated the simple mixing rule. 
(h) A void space exists (with a thickness of $\sim 5 \AA$, which is in the same order of magnitude as the equilibrium inter-molecular spacing values) between the surface of the nanoparticle and the solvent molecules in the compressed phase [36, 37]. However, the volume of the void space between the nanoparticle and the compressed phase was neglected in one of the studies [35]. The authors erroneously claimed that the void space increases with nanoparticle size (and to be as high as $1.3 \mathrm{~nm}$, which is higher than the equilibrium intermolecular spacing values). The correlation developed in this study implies that as the particle size increases the size of the compressed phase increases, which contradicts the idea of nanoparticles enhancing density by bigger margin than micron-scale particles. The plots for the comparison of the experimental data are also inconclusive as they occupy the region between predictions from the mixing rule and the predictions from the erroneous model developed by the authors.

(i) One study accounted for the effects of agglomeration (where the secondary nanostructures are expected to play a dominant role and would permeate the whole volume intervening the nanoparticle clusters) [34]. However, the authors did not consider previous reports in the literature, where electron microscopy images distinctively demonstrated the compressed layer occupying the intervening volume between clusters of closely spaced nanoparticles. Further, the authors also erroneously assumed that the compressed phase (or nanolayer) existed on the outskirts of the cluster and not within the cluster. This would cause the effect of the clusters to be underestimated by orders of magnitude. For density values exceeding $1100 \mathrm{~kg} / \mathrm{m}^{3}$, the authors report predicted values of density to the fourth place of decimal. As a result, the conclusions presented by the authors are circumspect and in need of additional verification.

The structure of this paper is as follows. Section 2 describes the analytical model, selection of solvent, nanoparticle candidates employed in this study. Section 3 outlines the details of experimental apparatus, and the results are discussed in Sect. 4. Finally, conclusions are presented in Sect. 5.

\section{Methodology}

\subsection{Analytical model}

Review of experimental results in the literature clearly demonstrate the deficiencies of the simple mixing rule for accurately predicting the density of nanofluids. In this context, a simple analytical model to predict the effective density of nanofluids accounting for the interfacial phenomena (at the solid-liquid interface) has been proposed in this study. The model proposed in the present study depicting the deviant densification of neat solvents is of practical relevance for the applications of nanofluids as coolants and for thermal energy storage (TES), including phase change materials (PCM) such as molten salt nanofluids, lubricants (due to their anomalous rheological properties: such as, shear-thinning and sometimes, shear-thickening behavior), industrial grade solvents, as well as for space travel and nuclear energy (e.g., for radiation mitigation and as radiation shields) and the shipment of densified hydrocarbons (e.g., in pipelines and shipping/ oil-tankers, such as, in the form liquid natural gas/LNG).

The simple mixing rule, based on the classical approach using statistical mechanics of ideal gas mixtures, has been commonly used by the researchers to predict the effective density of nanofluids [38]. This rule is solely based on the mass fraction of the constituents and does not consider the effect of particle dimensions, geometry (morphology and shape), size distribution and surface phenomena. According to classical mixing theory, effective density of a mixture $\left(\rho_{T 1}\right)$, consisting of a solvent phase and suspended solid particles, solely depends on the density of the pure solvent phase $\left(\rho_{f}\right)$, the density of the solid phase or nanoparticles $\left(\rho_{n}\right)$ and their mass fractions $(x)$; or the particle volume fraction $(\varphi)$. By using simple mixture rule (from thermodynamic equilibrium theory [39]), the effective values of density of the nanofluid $\left(\rho_{T 1}\right)$ can be expressed as a function of these three parameters, i.e., $\rho_{f}, \rho_{n}$ and $x(o r, \varphi)$.

A simple mathematical model accounting for the interfacial phenomena at the solid-liquid interface was implemented in this study. The model developed in this study is based on a simple schematic and was formulated by considering an ensemble of spherical nanoparticles suspended and dispersed uniformly (or homogeneously) in a liquid phase (solvent). As shown in Fig. 1, a compressed phase of the solvent molecules of a certain thickness $(\delta)$ was assumed to envelope each nanoparticle. An intervening "void space" ( $\varepsilon$, which is the same order of magnitude as the equilibrium spacing for the molecules due to intermolecular interactions, such as due to Van Der Waals forces and ionic repulsion interactions) was assumed to exist between the surface of the spherical nanoparticle and the compressed phase of solvent molecules that was assumed to completely envelope each nanoparticle (engulfing each nanoparticle). Hence, a compressed layer (with a nominal thickness, $\varepsilon$, of $1-2 \mathrm{~nm}$ ) of solvent molecules is considered to be the dominant player that modulates the effective density of the nanofluids (along with an intervening void space with a thickness, $\varepsilon$, of $\sim 0.5 \mathrm{~nm}$, that is modeled to 
Fig. 1 Representation of a spherical nanoparticle in a base fluid and surrounding interfacial layer
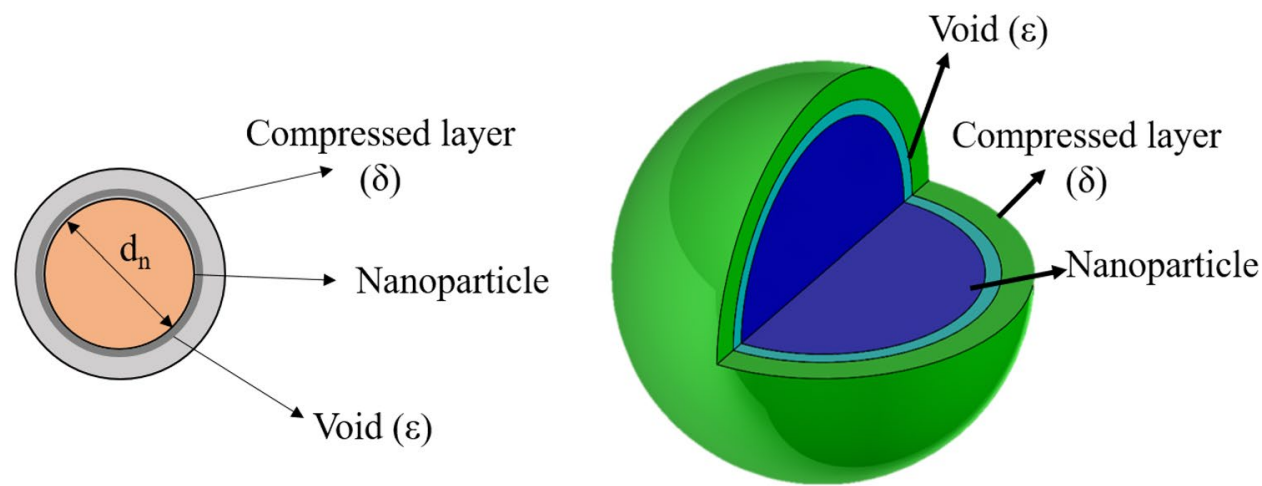

exist between the compressed phase and the surface of the nanoparticle).

For a spherical nanoparticle, the analytical model (termed as compressed phase model), accounts for the contribution of the elevated density of the compressed phase $\left(\rho_{c}\right)$ and the density of the bulk solvent phase $\left(\rho_{f}\right)$ (which also the density of the neat solvent), as well as the density of the nanoparticle $\left(\rho_{n}\right)$ to the total density of the nanofluid $\left(\rho_{T 2}\right)$. The total density of the mixture $\left(\rho_{T 2}\right)$ is calculated based on the mass fractions of the three individual components (nanoparticle, bulk solvent phase and compressed phase). The size and mass fraction of the compressed phase is varied parametrically (and this is based on information gleaned from the experimental measurements reported in the literature [40] for the thickness of the compressed phase and the numerical predictions reported in the literature $[6,7]$ for the density of the compressed phase estimated using Molecular Dynamics simulations). Based on this analytical model, the effective values of density for the nanofluid samples can be computed as:

$\frac{1}{\rho_{T 2}}=\frac{x}{\rho_{n}}+\frac{y}{\rho_{c}}+\frac{(1-x-y)}{\rho_{f}} ; v_{T 2}=x v^{n}+y v_{c}+(1-x-y)$

where, $\rho_{T 2}$ is the total (or effective) density (i.e., accounting for the compressed phase), $\boldsymbol{x}$ is the mass fraction of the nanoparticles, $\boldsymbol{y}$ is the mass fraction of the compressed phase, $\rho_{c}$ is the density of compressed phase, $\rho_{\boldsymbol{n}}$ is the density of a nanoparticle, and $\rho_{f}$ is the density of the bulk phase of the solvent (i.e., the density of the neat solvent), $\boldsymbol{v}_{T 2}$ is the total (or effective) specific volume (i.e., accounting for the compressed phase), $\boldsymbol{v}_{c}$ is the specific volume of compressed phase, $\boldsymbol{v}_{\boldsymbol{n}}$ is the specific volume of a nanoparticle, and $\boldsymbol{v}_{\boldsymbol{f}}$ is the density of the bulk phase of the solvent (i.e., the density of the neat solvent).

In contrast, using the simple mixing rule-the prediction from the conventional theory (which does not account for the formation of the compressed phase) can be used to predict the total (or effective) value of density of the nanofluid, $\rho_{T 1}$ (or specific volume, $v_{T 1}$ ) using a simple mixing rule and is expressed as follows:

$\frac{1}{\rho_{T 1}}=\frac{x}{\rho_{n}}+\frac{(1-x)}{\rho_{f}} ; v_{T 1}=x v_{n}+(1-x) v_{f}$

Rearranging the terms in Eq. (2), we have

$\rho_{T 1}=\frac{\rho_{f}}{x\left(\frac{\rho_{f}}{\rho_{n}}\right)+(1-x)}$

Defining,

$F_{1}=x\left(\frac{\rho_{f}}{\rho_{n}}\right)+(1-x)=1-x\left[1-\left(\frac{\rho_{f}}{\rho_{n}}\right)\right]$

the density, $\rho_{T 1}$, (or specific volume, $v_{T 1}$ ) predicted by simple mixing rule can be expressed as:

$F_{1}=x\left(\frac{\rho_{f}}{\rho_{n}}\right)+(1-x)=1-x\left[1-\left(\frac{\rho_{f}}{\rho_{n}}\right)\right]$

Similarly, rearranging the terms for Eq. (3), i.e., by accounting for the contribution of the compressed phase, the total density can be expressed as follows:

$\left(\frac{\rho_{f}}{\rho_{T 2}}\right)=x\left(\frac{\rho_{f}}{\rho_{n}}\right)+y\left(\frac{\rho_{f}}{\rho_{c}}\right)+(1-x-y) ;$

From purely geometric considerations-for a spherical nanoparticle, the ratio of the mass fractions for the nanoparticle $(x)$ and the bulk of the solvent phase $(y)$ can be expressed as:

$\left(\frac{y}{x}\right)=\frac{V_{c}}{V_{n}} \frac{\rho_{c}}{\rho_{n}}$

where, $V_{n}$ is the volume of a nanoparticle and $V_{c}$ is the net volume of the compressed phase (also, considering that there is zero mass in the void space). Accounting for the thickness of the compressed layer $(\delta)$ and the thickness of the void region $(\varepsilon)$ between the compressed phase and surface of the nanoparticle (which is also the average 
equilibrium spacing between the first layer of the solvent molecules and the surface of the nanoparticles), the relationship between $r_{c}$ (radial location of the outer extent of the compressed phase) and $r_{n}$ (radius of the spherical nanoparticle) can be expressed as:

$r_{c}=r_{n}+\varepsilon+\delta$

From Eq. (8), Eq. (6) is expressed as:

$$
\left(\frac{y}{x}\right)=\left(\frac{V_{c}}{V_{n}}\right)\left(\frac{\rho_{c}}{\rho_{n}}\right)=\left[\left(\frac{r_{c}}{r_{n}}\right)^{3}-\left(1+\frac{\varepsilon}{r_{n}}\right)^{3}\right]\left(\frac{\rho_{c}}{\rho_{n}}\right)
$$

Introducing the mass fraction of compressed phase from Eq. (9) in Eq. (6) results in

$$
\rho_{T 2}=\frac{\rho f}{F_{1}+\left(\frac{\rho_{f}}{\rho_{n}}\right) y-y}
$$

Defining,

$F_{2}=F_{1}-y\left[1-\left(\frac{\rho_{f}}{\rho_{c}}\right)\right]=F_{1}-x\left(\frac{\rho_{c}}{\rho_{n}}\right)\left[1-\left(\frac{\rho_{f}}{\rho_{c}}\right)\right]\left(\frac{V_{c}}{V_{n}}\right)$

the density predicted by the modified mixing rule (accounting for the contribution to the total density from the compressed phase) can be expressed as:

$\rho_{T 2}=\frac{\rho_{f}}{F_{2}} ; v_{T 2}=v_{f} F_{2}$

Hence the surplus contribution to density $\left(\rho_{T 21}\right)$, or specific volume $\left(v_{T 21}\right)$, that is above and beyond the predicted values for density that is obtained from the simple mixing rule, can be expressed as:

$\rho_{T 21}=\rho_{T 2}-\rho_{T 1}=\frac{\rho_{f}}{F_{2}}-\frac{\rho_{f}}{F_{1}} ; v_{T 21}=v_{T 2}-v_{T 1}=v_{f}\left(F_{2}-F_{1}\right)$

Hence, the percentage value of the surplus contribution to specific volume $\left(\Delta_{v 21}\right)$, that is above and beyond the predicted values for density obtained from the simple mixing rule, can be expressed as:

$\Delta_{v 21}=\frac{\left(v_{T 2}-v_{T 1}\right)}{v_{T 1}}=\frac{\left(F_{2}-F_{1}\right)}{F_{1}}$

Substituting for the values of $F_{2}$ and $F_{1}$, the following expression is obtained:
$\Delta_{v 21}=-\frac{x\left(\frac{\rho_{c}}{\rho_{n}}\right)\left[1-\left(\frac{\rho_{f}}{\rho_{c}}\right)\right]\left(\frac{V_{c}}{V_{n}}\right)}{1-x\left[1-\left(\frac{\rho_{f}}{\rho_{n}}\right)\right]} ;$
$\left(\frac{\Delta_{v 21}}{x}\right)=-\frac{\left(\frac{\rho_{c}}{\rho_{n}}\right)\left[1-\left(\frac{\rho_{f}}{\rho_{c}}\right)\right]\left(\frac{V_{c}}{V_{n}}\right)}{1-x\left[1-\left(\frac{\rho_{f}}{\rho_{n}}\right)\right]}$

Equation (15) shows that the absolute value of $\Delta_{v 21}$ is maximized (i.e., surplus density term or the "deviant" density term is enhanced) when the numerator is maximized and the denominator is minimized. Hence, the value of $\Delta_{v 21}$ is maximized, when:

1. the value of $\rho_{n}$ is minimized (i.e., nanoparticles should have low density: preferably, the specific gravity of the nanoparticles should be less than 1);

2. the value of $\rho_{c}$ is maximized (i.e., the compressed layer should have a high value of density, preferably, the specific gravity of the neat solvent should be more than 1; as a result aqueous nanofluids are ruled out since, as the compressed phase formed by water molecules on nanoparticle surface are expected to possess properties similar to that of ice; and ice typically has a specific gravity less than 1 );

3. the value of $\rho_{f}$ is minimized (i.e., the neat solvent should have a low density: preferably, the specific gravity of the neat solvent should be less than 1);

4. the ratio of $\left(\frac{\rho_{f}}{\rho_{c}}\right)$ should be less than 1 (i.e., the solid phase of the solvent should have a higher density than the liquid phase of the solvent; as a result-aqueous nanofluids are ruled out-as the compressed phase formed by water molecules on nanoparticle surface are expected to possess properties similar to that of ice; and since ice typically has a specific gravity less than 1);

5. the volume of the compressed phase is maximized (i.e., the volume of the compressed phase should be as high as possible);

6. the volume (or size) of the nanoparticle should be minimized (i.e., the volume or size of the nanoparticle should be as low as possible).

This theoretical analysis (for $\Delta_{v 21}$ and $\rho_{T 21}$ ) shows that careful design of experiments is needed to be able to conclusively discern the deviant enhancement in density arising from the contribution of the compressed phase while every effort should be made for minimizing measurement uncertainty. 


\subsection{Selection of solvent candidates}

The results from the MD simulations [7] show that the density of the compressed phase is typically $25-100 \%$ higher (i.e., almost double) than that of the neat of solvent (especially for non-aqueous solvents, such as for organic solvents, particularly for oil-based solvents and molten salt eutectics). Considering this behavioral pattern reported in the literature involving MD simulations, in this study, oil based solvents were chosen for the experiments. A paraffin-based oil, which is typically used commercially as phase change material (PCM), was procured for this study (from PureTemp, with commercial brand name: PureTemp-15X). Hence, assuming that for oil-based solvents, approximately, $\left[\left(\frac{\rho_{c}}{\rho_{f}}\right)=2\right]$; we get:

$\Delta_{v 21}=-\frac{x\left(\frac{\rho_{c}}{\rho_{n}}\right)\left(\frac{v_{c}}{V_{n}}\right) A}{1-x\left[1-\left(\frac{\rho_{f}}{\rho_{n}}\right)\right]}$

where, the correction factor, $A$, is approximately 0.5 for oils. In contrast, for water, approximately, $\left[\left(\frac{\rho_{c}}{\rho_{f}}\right) \approx 0.9\right][9]$; since ice has a specific gravity of 0.9 . As a result, we get, a positive value of $\Delta_{v 21}$, as shown below:

$\Delta_{V 21}=-\frac{x\left(\frac{\rho_{c}}{\rho_{n}}\right)\left(\frac{V_{c}}{V_{n}}\right) B}{1-x\left[1-\left(\frac{\rho_{f}}{\rho_{n}}\right)\right]}$

where, the correction factor, $\mathrm{B}$, is approximately 0.1 .

This theoretical analysis shows that the detection of the deviant density (or surplus density) caused by the compressed layer is virtually undetectable in experiments involving aqueous nanofluids and the density of the aqueous nanofluids are more likely to conform to the simple mixing rule (even for nanoparticles less than $6 \mathrm{~nm}$ diameter). Hence, aqueous nanoparticles may have a miniscule deviation in density (i.e., miniscule decrease in density) than that of the values predicted by the simple mixing rule. This deviant density reduction for aqueous nanofluids is so tiny (estimated to be less than $\sim 0.5 \%$ ) that it is virtually undetectable by most of the conventional measurement techniques or commercial instruments that are typically used for measuring density (e.g., using densitometers). However, this analyses has significant implications for the specific heat capacity of aqueous nanofluids as well. This also implies, that the detection of the deviant values of specific heat capacity (or surplus values of specific heat capacity) caused by the compressed layer is virtually undetectable in experiments involving aqueous nanofluids and the specific heat capacity of the aqueous nanofluids are more likely to conform to the simple mixing rule and there may be a miniscule degradation (less than $2 \%$ ) in the values of specific heat capacity of aqueous nanofluids (even for nanoparticles less than $6 \mathrm{~nm}$ diameter), that are very difficult to detect since the typical measurement uncertainty for measurement of specific heat capacity (e.g., using Differential Scanning Calorimetry/ DSC) is approximately $5 \%$ or more. As mentioned before, it is interesting to note that studies in the literature (e.g., [33]) showed degradation in the specific heat capacity of aqueous nanofluid samples and were consistently below the predictions afforded by the various flavors of the simple mixing rule.

\subsection{Selection of nanoparticle candidates}

Apriori, it is not known, what the density or thickness of the compressed phase will be for a given nanofluid (e.g., only indirect estimates can be obtained for these parameters from MD simulations), since these parameters are also very sensitive to even minute variations in the synthesis conditions (e.g., $\mathrm{pH}$, surfactant concentration, synthesis temperature, etc.). Hence, to begin with, the density of the nanoparticles should be minimized (as the primary control variable in these experiments).

However, this creates a conundrum, since the conventional nanoparticles that are typically used for nanofluids experiments in the literature (such as metallic, ceramic or carbon-based nanoparticles) have specific gravity values typically exceeding 2 [41]. Typically, ceramic nanoparticles (such as silica, alumina, etc.) have a specific gravity of 2 (approximately). Metallic nanoparticles (such as tungsten, gold, silver, etc.) possess even higher values of specific gravity, often exceeding 2. Carbon based nanoparticles also possess specific gravity typically exceeding 2 (e.g., 2.2 for graphene, 3.5 for exfoliated graphite, 1.4 for carbon nanotubes, 3.4 for diamond nanoparticles, etc.). Carbon nanotubes have the lowest specific gravity but are also very difficult to disperse in typical solvents with the goal of obtaining stable nanofluids, i.e., for either aqueousnanofluids or oleo-nanofluids (oil based nanofluids).

A brief literature search reveals that organic nanoparticles (such as proteins, lipids/ vesicles, etc.) have specific gravity less than 1 (typically, the values range from 0.4 to 0.9) [42]. However, commercial procurement of these nanoparticles tend to be very expensive and the material (or chemical) stability is very unreliable as proteins (as well as lipids/ vesicles) tend to denature promptly when subjected to thermal shock or mechanical stresses / shear forces or chemical gradients.

Interestingly, the proteins obtained from dehydrated non-fat skimmed milk (which is primarily composed of the protein, casein) are stable (even for severe thermal shock) 
and are easy to dissolve in water or oil. The Stokes-diameter of casein (as typical of various proteins and peptides) is expected to range from 2 to $6 \mathrm{~nm}$. The specific gravity of this class of proteins (e.g., casein) varies from 0.45 to 0.5 [42]. Hence, protein suspensions created using casein, were targeted in this study (i.e., sourced from dehydrated non-fat skimmed milk).

Based on the predictions from the analytical model, the experimental validation performed in this study was targeted for protein suspensions in oil, i.e., oleo-nanofluids (since aqueous nanofluids are unsuitable due to the marginal deviations in the density compared to that of the predictions afforded by the simple mixing rule and these deviations are virtually undetectable owing to the deviations being less than the typical values of measurement uncertainty). In contrast, oleo-nanofluids with low density proteins (such as low density lipo-proteins/ LDL) is expected to provide sufficient resolution for discerning the "deviant" component of the density enhancement such that the experimental results can be used to conclusively differentiate the magnitude of the surplus density that is higher than the values of the measurement uncertainty in these experiments.

The neat solvent chosen for this study was an oil (PureTemp-15X). Casein was chosen as the ideal low-density nanoparticle candidate (since it has specific gravity less than 1) for ensuring the success of the experimental measurements performed in this study. As mentioned before, the Stokes-diameter of the protein used in this study is expected to range from 2 to $6 \mathrm{~nm}$ [42] (depending on the size of the complexes formed between surfactants and proteins along with differing levels of hydration for the hydrated-protein molecules). The synthesis protocol, experimental apparatus and procedure as well as the results (both analytical predictions and experimental validation) are discussed next.

\section{Experimental apparatus and procedure}

\subsection{Synthesis protocol}

The oleo-nanofluid samples explored in this study are obtained by mixing the protein nanoparticles (casein) in a paraffin-based oil (procured under the commercial name PureTemp-15X). The following synthesis protocol, based on prior reports in the literature [43-45], was established for the preparation of the protein-based oleo-nanofluid samples:
Step 1 Add surfactant (Sodium Dodecyl Sulfate or "SDS") to water (for example at mass concentration of $0.5 \%)$.

Step 2 Dissolve milk powder in small batches in a cold aqueous solution of surfactant by manual stirring.

Step 3 Add surfactant (SDS) to oil (neat solvent) and stir gently.

Step 4 Mix the aqueous solution of milk (from Step 2) and the oil solution (from Step 3) while stirring manually.

Step 5 Fill the Ultra-sonicator with ice cubes and water alongside salt to maintain sub-zero temperature in the bath.

Step 6 Ultra-sonicate the whole mixture for about $30 \mathrm{~min}$ Step 7 Use a pipette to extract the oleo-nanofluid samples and measure density.

The detailed specification for the mass of the ingredients used in this synthesis protocol are listed in Table 1.

It should be noted that the specific gravity of SDS is typically 1.01 [46] while the Stokes-diameter of SDS is typically $\sim 1 \mathrm{~nm}$ [47]. In addition, as mentioned before, the Stokes-diameter of the hydrated-protein-SDS complex is expected to range from 2 to $6 \mathrm{~nm}$ [42].

\subsection{Experimental apparatus}

The apparatus for measuring the density of the nanofluid samples in this study was based on the Archimedean principle of buoyancy of immersed bodies along with an unequal-arm balance. A wire attached to one end of the balance arm (lever) was used to suspend a "sinker" of a known standard mass. In this technique, the density of an unknown liquid was calculated from the buoyancy force exerted by the liquid on the immersed "sinker" (calibrated value of the standard mass). This experimental apparatus enables the measurement of the buoyancy force by measuring the difference in weight for the sinker immersed in the test fluid (i.e., nanofluid with unknown density) compared to that of the baseline state (i.e., the mass of the sinker in air). All

Table 1 Mass of ingredients used for the synthesis of samples of oleo-nanofluid

\begin{tabular}{lll}
\hline Beaker & Component & Mass \\
\hline Water solution & Water & $10 \mathrm{~g}$ \\
& SDS & $0.05 \mathrm{~g}$ \\
Oil solution & NaOH & $0.1 \mathrm{~g}$ \\
& Oil (PCM) & $10 \mathrm{~g}$ \\
& SDS & $0.05 \mathrm{~g}$ \\
& Milk Powder & $0.1 \mathrm{~g}$ \\
\hline
\end{tabular}


Fig. 2 Experimental apparatus for measuring the density of nanofluids

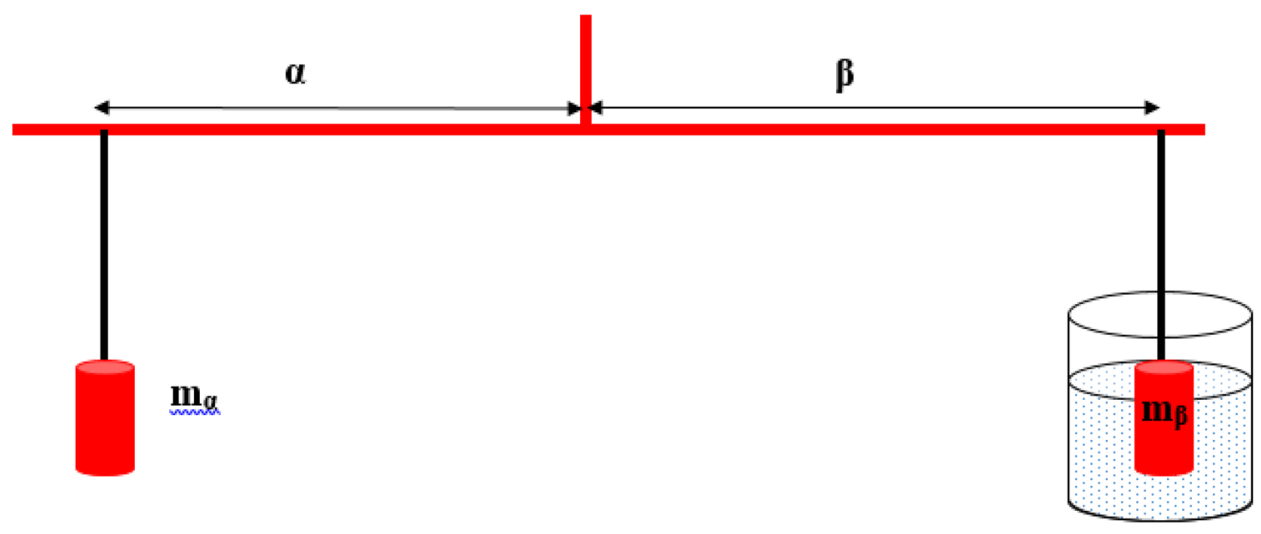

measurements were performed under ambient conditions (i.e., at a room temperature of $24^{\circ} \mathrm{C}$ ) (Fig. 2).

$m_{\beta}^{\text {liquid }}=\frac{m_{\alpha} \cdot \alpha}{\beta}$

$\rho_{\text {liquid }}=\frac{\rho_{\text {SS,mass }} *\left(m_{\beta}^{\text {air }}-m_{\beta}^{\text {liquid }}\right)}{m_{\beta}^{\text {air }}}$

where, $m_{\text {air }}$ is the mass of the body immersed in air, $m_{\text {liquid }}$ is the mass of the body in liquid (reduced due to buoyancy), $\boldsymbol{\rho}_{\text {liquid }}$ is the density of the test liquid, $\boldsymbol{\rho}_{s s, \text { mass }}$ is the density of the sinker (which is the same as the density of stainless steel).

\subsection{Uncertainty analysis}

Uncertainty values of the experimental measurements were quantified using the Kline-McClintock method [11]. The measurement uncertainty associated with the technique developed in this study is described below.
- Let $\Delta a$ and $\Delta \beta$ be the uncertainties in the length measurement (of $a$ and $\beta$, respectively). The least count of the scale is $0.125 \mathrm{in}$. The uncertainty in the measurement is $50 \%$ of least count. Therefore, $\Delta a=\Delta \beta=0.0625$ in.

- The counter masses used in the experiment are standard mass (NIST calibrated) and the uncertainty in the measurement can be neglected (since the differential change in the measurement of mass is required in these experiments).

- The density of stainless steel is obtained from the NIST data base [48].

\section{Results and discussion}

Before measuring the density of nanofluids, the experimental apparatus is calibrated by measuring the density of pure liquids whose densities are well known, namely mineral oil and DI water. The density measurements were performed for both liquids at a temperature of $24^{\circ} \mathrm{C}$. Table 2 summarizes the results from the benchmark tests

Table 2 Experimental measurements for samples of oleo-nanofluids: asein based protein nanoparticles and SDS surfactant dispersed in PureTemp-15X (Baseline Density for Neat Solvent, $\rho_{\mathrm{f}}=860\left[\left(\mathrm{~g} / \mathrm{m}^{3}\right)\right.$; Measurement Uncertainty $<1 \%$ )

\begin{tabular}{|c|c|c|}
\hline$a \pm \Delta a$ & $12.375 \pm 0.0625$ (inches) & \\
\hline$\beta \pm \Delta \beta$ & $14.125 \pm 0.0625$ (inches) & \\
\hline $\begin{array}{l}m_{a} \\
\text { sinker mass in air }\end{array}$ & $20(g)$ & \\
\hline $\begin{array}{l}m_{\beta} \\
\text { for sinker completely immersed in sample (oleo-nanfluid) }\end{array}$ & $17.52(\mathrm{~g})$ & \\
\hline $\begin{array}{l}\rho_{S S} \\
\text { Density of stainless steel }\end{array}$ & $7700\left(\mathrm{~kg} / \mathrm{m}^{3}\right)$ & \\
\hline $\begin{array}{l}\rho_{\mathrm{T} 2} \\
\text { Measured value of density for the sample (oleo-nanofluid) }\end{array}$ & $953.9\left(\mathrm{~kg} / \mathrm{m}^{3}\right)$ & $12.2 \%$ (enhancement over $\rho_{f}$ ) \\
\hline $\begin{array}{l}\rho_{T 1} \\
\text { Predicted value using simple mixing rule }\end{array}$ & $924.4\left(\mathrm{~kg} / \mathrm{m}^{3}\right)$ & $8.8 \%$ (enhancement over $\rho_{f}$ ) \\
\hline $\begin{array}{l}\rho_{T 21}=\rho_{T 2}-\rho_{T 1} \\
\text { Surplus Density }\end{array}$ & $29.4\left(\mathrm{~kg} / \mathrm{m}^{3}\right)$ & $3.2 \%$ (enhancement over $\rho_{T 1}$ ) \\
\hline
\end{tabular}


Table 3 Benchmark test results for DI Water and Mineral Oil

\begin{tabular}{llllll}
\hline $\begin{array}{l}\text { Test } \\
\text { liquid }\end{array}$ & $\begin{array}{l}\text { Mass in } \\
\text { air }(\mathrm{g})\end{array}$ & $\begin{array}{l}\text { Mass in } \\
\text { liquid } \\
(\mathrm{g})\end{array}$ & $\begin{array}{l}\text { Density } \\
\text { of liquid } \\
\left(\mathrm{kg} / \mathrm{m}^{3}\right)\end{array}$ & $\begin{array}{l}\text { Standard } \\
\text { value } \\
{[48] \text { of }} \\
\text { density } \\
\left(\mathrm{kg} / \mathrm{m}^{3}\right)\end{array}$ & $\begin{array}{l}\text { Difference } \\
(\%)\end{array}$ \\
\hline $\begin{array}{l}\text { DI Wwa- } \\
\text { ter }\end{array}$ & 20 & 17.3 & 1022.6 & 1000 & 2.2 \\
$\begin{array}{c}\text { Mineral } \\
\text { oil }\end{array}$ & 20 & 17.8 & 842.1 & 850 & 0.9 \\
\hline
\end{tabular}

(calibration tests) that were performed with DI water and mineral oil. The results from the experimental measurements show that the percentage difference between the measured values and the values listed in the ASTM standard database are: $2.2 \%$ and $0.9 \%$, for DI water and mineral oil, respectively (hence, for measurements involving oil based samples, the measurement uncertainty is expected to be less than 1\%). Hence, the overall uncertainty for the density ratio between the test samples and reference samples ranged from $0.9 \%$ 1\% (based on a minimum of three repeats for each test). After performing the benchmark tests (calibration tests), the density measurement of the samples of oleo-nanofluids were performed. The results of the measurements are presented in Table 3.

As the experimental results show, the traditional mixing rule under predicts the density of nanofluid by $3.2 \%$. The value of density measured through Archimedean technique for the synthesized nanofluid is $953.9 \mathrm{~kg} / \mathrm{m}^{3}$ while the traditional mixing rule predicts the density of the nanofluid to be $924 \mathrm{~kg} / \mathrm{m}^{3}$. The density of the neat solvent is $860\left(\mathrm{~kg} / \mathrm{m}^{3}\right.$ ) (results provided in Appendix).

To ascertain the proportion of the contribution of the compressed phase to the total density of the nanofluid samples in these experimental results, the predictions from the analytical model were obtained by parametric variation of both the nanoparticle size and the thickness of the compressed phase. The predictions from the analytical model are plotted in Fig. 3. The specific gravity of the compressed phase was assumed to be the same as that of the solid phase of the solvent $\left[\rho_{c}=950\left(\mathrm{~kg} / \mathrm{m}^{3}\right)\right]$ while the specific gravity of the liquid phase of the solvent is assumed to be the same as that of the neat solvent at $24^{\circ} \mathrm{C}\left[\rho_{f}=860\right.$ $\left.\left(\mathrm{kg} / \mathrm{m}^{3}\right)\right]$. The plots are obtained by assuming $\varepsilon=5 \AA$ (i.e., $0.5 \mathrm{~nm})$. The plots are obtained for two different values of the compressed layer thickness $(\delta)$, i.e., $\delta=1 \mathrm{~nm}$ and $\delta=2 \mathrm{~nm}$. The plots show that for nanoparticle radius of $2 \mathrm{~nm}$ the surplus density is $3 \%$ (for $\delta=1 \mathrm{~nm}$ and $x=0.5 \%$ ) while the value of surplus density is $6 \%$ (for $\delta=2 \mathrm{~nm}$ and $x=0.5 \%)$. Hence, correlating the experimental measurements to the numerical predictions, it is expected that the thickness of the compressed phase engulfing the protein
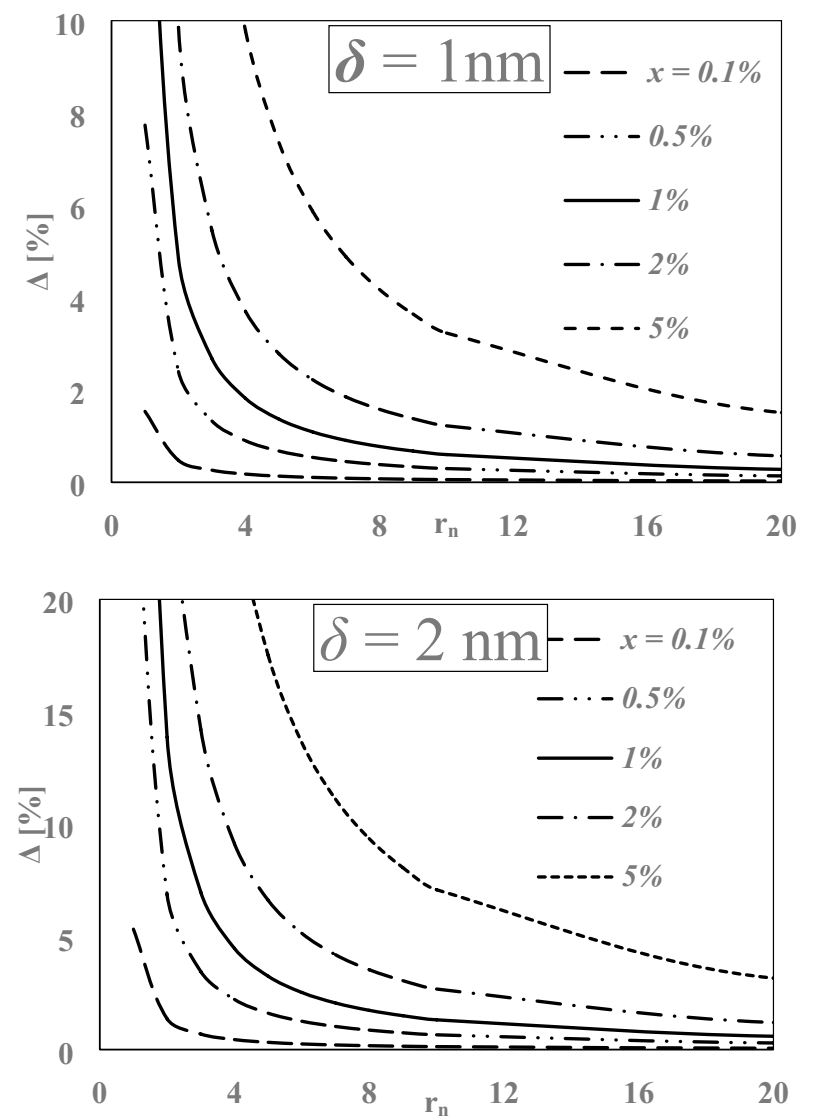

Fig. 3 Plots for analytical predictions for estimating the magnitude of density surplus $(\Delta)$ in oleo-nanofluid suspension and as a function of the Stokes-radius of a nanoparticle (e.g., casein/ milk proteins dispersed in a PCM oil) at different mass fractions of the protein nanoparticles. The deviant density enhancement (i.e., surplus density) is hypothesized to occur due to the presence of a compressed phase that forms on the surface of a nanoparticle and in turn engulfs each casein nanoparticle within the bulk phase of the oil (PCM)

nanoparticles (casein) is approximately in the range of $1-2 \mathrm{~nm}$.

Further, the model developed in this study also shows that this phenomenon, detection of surplus density, is virtually impossible for aqueous nanofluids since the specific gravity of the compressed phase within water, being structurally akin to that of ice, is less than 1 . The analytical model developed in this study also shows that this phenomenon is probably more difficult for conventional nanoparticles, such as metallic and ceramic nanoparticles, since their values of specific gravity are typically 2 or higher (which damps down the density enhancement terms in Eq. 16). The model involves the relative contribution from the three component phases: (1) the bulk solvent phase; (2) nanoparticle; and (3) the compressed phase formed within the solvent that engulfs each nanoparticle. 
To conclude, the predictions from the analytical model shows that the density surplus can be higher for smaller nanoparticles as well as for higher mass fractions of protein nanoparticles. The surplus density is higher for nanoparticles with smaller density than the fluid (solvent phase). The predictions from the analytical model developed in this study show that with proper optimization of the experimental parameters and the synthesis conditions, the surplus density (deviant density enhancement) can exceed $10 \sim 20 \%$ for mass fraction of $1 \%$ for the protein nanoparticles (as well as for other types of low-density nanoparticles such as lipids/ vesicles and other low density lipo-proteins/ "LDL").

\section{Conclusions}

In the present study, the predictions for the magnitude of surplus density of a protein suspension in oil (oleonanofluid), due to compressed phase of solvent engulfing the nanoparticle, were validated experimentally. This phenomenon (surplus density) is caused by the surface adsorption of the solvent molecules on the nanoparticle surface and, in turn, can lead to the formation of secondary nanostructures (for a narrow range of parameters in certain circumstances) and is a manifestation of the "nanoFin Effect (nFE). The experimental results show that the conventional simple mixing rule approach underestimate the density by $3.2 \%$ (with a measurement uncertainty of less than 1\%). The experimental measurements were compared with that of the analytical predictions for the magnitude of the density surplus. The excess density measured in the experiments can be conclusively attributed to be caused by the presence of a compressed layer with a thickness of approximately $\sim 1 \mathrm{~nm}$. Furthermore, in this study we introduce a new strategy for optimizing and enhancing the material properties of non-aqueous nanofluids. The novel approach involves the use of nanoparticles (such as proteins, lipo-proteins, lipids/ vesicles, etc.) that have specific gravity less than 1 . For development of a sophisticated model to predict the effective density of nanofluids, it is worth investigating the molecular structure or morphology of the compressed layer around the nanoparticle for accurate determination of material properties of adsorbed compressed layer. Furthermore, a natural extension of this study would be to measure the density of different nanofluids with different combinations of solvent, and nanoparticle.

\section{Compliance with ethical standards}

Conflict of interest The authors declare that they have no conflict of interest.

\section{References}

1. Ma B, Kumar $n$, Kuchibhotla A, Banerjee D (2018) Experimental measurement of the effect of particle concentration on the specific heat capacity of silica nanofluids. In: IEEE Intersociety conference on thermal and thermomechanical phenomena in electronic systems (ITherm), San Diego.

2. Jo B, Banerjee $D(2011)$ Interfacial thermal resistance between a carbon nanoparticle and molten salt eutectic: effect of material properties, particle shapes and sizes. In: ASME/JSME 8th thermal engineering joint conference, Honolulu.

3. Lee J, Kuchibhotla A, Banerjee D, Berman D (2019) Silica nanoparticles as copper corrosion inhibitors. Mater Res Express 6(8):0850e3.

4. Banerjee D (2013) Guest eitorial for the special issue on micro/ nanoscale transport phenomena. J Nanotechnol Eng Med 3(3).

5. Oh S, Kauffmann Y, Scheu C, Kaplan W, Ruhle M (2005) Ordered liquid aluminum at the interface with sapphire. Science 310:661-663

6. Huisman WJ, Peters JF, Derks JW, Abernathy DL, van der Veen JF (1997) A new X-ray diffraction method for structural investigation of solid-liquid interfaces. Sci Instruments 11:4169

7. Jo B (2012) Numerical and experimental investigation of organic nanomaterials for thermal energy storage and for concentrating solar power applications. Texas A\&M University, College Station

8. Shin D, Banerjee D (2015) Enhanced thermal properties of $\mathrm{SiO} 2$ nanocomposite for solar thermal energy storage applications. Int J Heat Mass Transf 84:898-902

9. Shin D, Banerjee D (2011) Enhanced specific heat of silica nanofluid. J Heat Transf 133(2):024501

10. Tiznobaik H, Banerjee D, Shin D (2015) Effect of formation of long range secondary dendritic nanostructures in molten salt nanofluids on the values of specific heat capacity. Int J Heat Mass Transf 91:342-346

11. Binjian M, Banerjee D (2019) Numerical modelling of nanofluid thermal conductivity: the effect of nano-network on thermal transport behavior. J Heat Transf 141(12):122401

12. Singh N, Banerjee D (2013) Nanofins: science and applications. Springer, College Station

13. Sriraman SR (2007) Pool boiling on nano-finned surfaces. Texas A\&M University, College Station

14. Sathyamurthi V, Ahn HS, Banerjee D, Lau SC (2009) Subcooled pool boiling experiments on horizontal heaters coated with carbon nanotubes. J Heat Transf 131.

15. Zhong H, Lukes JR (2006) Interfacial thermal resistance between carbon nanotubes: Molecular dynamics simulations and analytical thermal modeling. Phys Rev B 74.

16. Estrada D, Pop E (2011) Imaging dissipation and hot spots in carbon nanotube network transistors. Appl Phys Lett 98.

17. Pollack GL (1969) Kaptiza resistance. Rev Mod Phys 41(1).

18. Singh N (2010) Computational analysis of thermo-fluidic characteristics of a carbon nano-fin. Texas A\&M University, College Station

19. Maune $\mathrm{H}, \mathrm{Chiu} \mathrm{HY}$, Bockrath $\mathrm{M}$ (2006) Thermal resistance of the nanoscale constrictions between carbon nanotubes and solid substrates. Appl Phys Lett 89.

20. Murad S, Puri IK (2008) Thermal transport across nanoscale solid-fluid interfaces. Appl Phys Lett 92. 
21. Huxtable ST, Cahill DG, Shenogin S, Xue L, Pzisik R, Barone P, Usrey M, Strano MS, Siddons G, Shim M (2003) Interfacial heat flow in carbon nanotube suspensions. Nature 2:731-734

22. Jung S (2012) Numerical and experimental investigation of inorganic nanomaterials for thermal energy storage (TES) and concentrated solar power (CSP) applicationss. Texas A\&M University, College Station

23. Shin D (2011) Molten salt nanomaterials for thermal energy storage and concentrated solar power applications. Texas A\&M University, College Station

24. Yang $\mathrm{H}$ (2014) Experimental and numerical investigation of pool boiling heat transfer on. Texas A\&M University, College Station

25. Oh SH, Kauffmann Y, Scheu C, Kaplan WD, Ruhle M (2005) Ordered liquid aluminum at the interface with sapphire. Science 310(5748):661-663

26. Li L, Zhnag Y, Ma H, Yang M (2010) Molecular dynamics simulation of effect of liquid layering around the nanoparticle on the enhanced thermal conductivity of nanofluids. J Nanopart Res 12(3):811-821

27. Chang $\mathrm{Cl}$, Lee, WJ, Young TF, Ju SP, Chang CW, Chen HL, Chang JG (2008) Adsorption mechanism of water molecules surrounding Au nanoparticles of different sizes. J Phys Chem 128(15).

28. Jo B, Banerjee D (2015) Effect of solvent on specific heat capacity enhancement of binary molten salt-based carbon nanotube nanomaterials for thermal energy storage. Int J Thermal Sci 98:219-227

29. Singh N, Banerjee D (2013) Nanofins: science and applications. Springer, Heidelberg

30. Vajjh RS, Das DK, Mahagaonkar BM (2009) Density measurement of different nanofluids and their comparison with theory. Pet Sci Technol 27(6):612-624

31. Mariano A, Pastoriza-Gallego MJ, Lugo L, Camacho A, Canzonieri S, Pineiro M (2013) Thermal conductivity, rheological behaviour and density of non-Newtonian ethylene glycol-based $\mathrm{SnO}_{2}$ nanofluids. Fluid Phase Equilibria, pp. 119-124.

32. Pastoriza-Gallego MJ, Casanova CJ, Paramo R, Barbes B, Legido JL, Pineiro MM (2009) Study on stability and thermophysical properties (density and viscosity) of $\mathrm{Al} 2 \mathrm{O} 3$ in water nanofluid. J Appl Phys.

33. Heyhat MM, Kowsary F, Rashidi AM, Momenour MH, Amrollahi A (2013) Experimental Investigation of laminar convective heat transfer and pressure drop of water-based $\mathrm{Al} 2 \mathrm{O} 3$ nanofluids in fully developed flow regime. Exp Thermal Fluid Sci 44:483-489

34. Selvakumar DR, Wu J (2019) A comprehensive model for effective density of nanofluids based on particle clustering and interfacial layer formation. J Molecular Liquids 292.
35. Sharifpur M, Yousefi S, Meyer PJ (2016) A new model for density of nanofluids including nanolayer. Int Commun Heat Mass Transfer 78:168-174

36. Yu CJ, Richter AG, Datta A, Durbin MK, Dutta P (1999) Observation of molecular layering in thin liquid films using $X$ ray reflectivity. Phys Rev Lett, pp. 2326-2329.

37. Yu CJ, Richter AG, Kmetko J, Dugan SW, Datta A, Dutta P (2001) Structure of interfacila liquids: $X$-ray scattering studies. Phys Rev E, p 021,205.

38. Choi SUS, Eastman JA (1995) Enhancing thermal conductivity of fluids with nanoparticles. In: 1995 International mechanical engineering congress and exhibition, San Francisco.

39. University of Cambridge [Online]. Available: https://www.doitp oms.ac.uk/tlplib/bones/derivation_mixture_rules.php. Accessed 24082019.

40. Feibelman PJ (2010) The first wetting layer on a solid. Phys Today 63(2).

41. [Online] Available: https://www.azonano.com/article.aspx?Artic lelD $=3398$

42. Pugliese A, Cabassi G, Chiavaro E, Paciulli M, Carini E, Mucchetti G. Physical characterization of whole and skim dried powders.

43. Saporito F, Sandri G, Bonferoni CM, Rossi S, Boselli C, Cornaglia IA, Mannucci B, Grisoli P, Vigani B, Ferrari F (2018) Essential oilloaded lipid nanoparticles for wound healing. Int J Nanomed, pp 175-186.

44. Guo M, Wang G (2016) Milk protein polymer and its application in environmentally safe adhesives. Polymers.

45. Zhnag L, Hayes D, Chen G, Zhong Q (2013) Transparent dispersions of milk-fat-based nanostructured lipid carriers for delivery of carotene. J Agric Food Chem 61:9435-9443

46. Duplatre G, Ferreira Marques MF, Graca MM (1996) Size of sodium dodecyl sulfate micelles in aqueous solutions as studied by positron annihilation lifetime spectroscopy. J Phys Chem 100:16608-16612

47. Reynolds JA, Tanford C (1970) The gross conformation of protein-sodium dodecyl sulfate complexes. J Biol Chem 245(19):5161-5165.

48. NIST Reference Tables. Available: https://www.nist.gov/ncnr/ sample-environment/sample-mounting/reference-tables

49. Singh N, Sathyamurthy V, Peterson W, Arendt J, Banerjee D (2010) Flow boiling enhancement on a horizontal heater using carbon nanotube coatings. Int J Fluid Flow 31: 201-207.

Publisher's Note Springer Nature remains neutral with regard to jurisdictional claims in published maps and institutional affiliations. 